\title{
Anthropologie et Iconologie
}

Réflexions sur les apports de Jack Goody à l'analyse de la notion de 'représentation'

\section{Daniel Russo}

\section{(2) OpenEdition}

\section{Journals}

\section{Édition électronique}

URL : https://journals.openedition.org/cem/4242

DOI : $10.4000 /$ cem.4242

ISSN : 1954-3093

Éditeur

Centre d'études médiévales Saint-Germain d'Auxerre

\section{Référence électronique}

Daniel Russo, « Anthropologie et Iconologie », Bulletin du centre d'études médiévales d'Auxerre / BUCEMA [En ligne], Histoire de l'art \& Anthropologie, mis en ligne le 27 février 2008, consulté le 24 septembre 2022. URL : http://journals.openedition.org/cem/4242 ; DOI : https://doi.org/10.4000/cem.4242

Ce document a été généré automatiquement le 24 septembre 2022.

\section{(c) $)$ (i) (2)}

Creative Commons - Attribution - Pas d'Utilisation Commerciale - Partage dans les Mêmes Conditions 4.0 International - CC BY-NC-SA 4.0

https://creativecommons.org/licenses/by-nc-sa/4.0/ 


\title{
Anthropologie et Iconologie
}

\author{
Réflexions sur les apports de Jack Goody à l'analyse de la notion de \\ 'représentation'
}

\section{Daniel Russo}

1 En rapport avec la problématique définie pour notre 'Séminaire', Iconography/Iconology Today $^{1}$, je me propose de relire l'ouvrage de Jack Goody (1919- ) sur la peur des 'représentations' et l"ambivalence' à l'égard des images, en le replaçant dans l'ensemble de son œuvre mais aussi en le situant plus largement dans les études portant sur l'anthropologie et l'iconologie envisagées dans leurs relations dynamiques, c'est-à-dire à deux niveaux principaux :- celui du statut des représentations iconiques ; - celui des travaux d'anthropologie des pratiques symboliques. Au cours de l'analyse, les hypothèses comme les remarques de Jack Goody rencontrent souvent celles de Nelson Goodman (1906-1998) sur le style et surtout, à propos de ce qui peut 'faire de l'art'2, sur la symbolisation dans "l'œuvre représentationnelle "; plus souvent encore les hypothèses de Hans Belting (1935- ) sur l'anthropologie des images dans ses rapports constitutifs au corps et à la mort ${ }^{3}$. J'adopterai pour fil conducteur de l'étude le concept, omniprésent chez Jack Goody, de la 'cognition' et celui, connexe, des 'contradictions cognitives', tous deux tout à fait pertinents pour examiner l'articulation fondamentale entre les rapports de l'analyse en iconographie comme en iconologie. Les différentes recherches de Jack Goody visent à circonscrire les effets différentiels des modes de communication, oraux ou écrits, sur la religion, le commerce, le droit, la science, l'organisation de l'Etat ou encore la littérature, comme structure ouverte et interactive avec les autres structures du social. J'insisterai sur ce que sa définition de la notion de 're-présentation', à travers le concept des contradictions cognitives, apporte à la compréhension du champ iconique et à la définition des tensions internes au champ mimétique 4 .

2 Je soulignerai ainsi trois points à travers mon approche:

- la formation de la notion des processus cognitifs dans l'œuvre de Jack Goody ;

- le processus de re-présentation et les contradictions cognitives qu'il inclut ;

- la pertinence iconologique du concept des 'contradictions cognitives', par rapport notamment à l'approche iconologique d'Erwin Panofsky (1892-1968) ${ }^{5}$. 


\section{La formation de la notion des processus cognitifs dans l'œuvre de Jack Goody}

3 Paru en 1997, le livre de Jack Goody doit être situé dans le contexte de ses quatre ouvrages majeurs qui développèrent et précisèrent les grandes thématiques d'une anthropologie cognitive toujours selon une visée comparatiste. A savoir, par ordre de parution :

Literacy in Traditional Societies ${ }^{6}$, recueil d'études anthropologiques et historiques sur la Somalie, Madagascar, la Chine ancienne, l'Angleterre pré-industrielle, où se trouve précisé le concept de 'littératie' (literacy) et ses conséquences sous l'intitulé, reconnu depuis, de la «technologie de l'intellect $»^{7}$. Par literacy (littératie), Jack Goody entend désigner l'ensemble de la praxis et des représentations liées à l'écrit, depuis les conditions matérielles de la réalisation effective (supports et outils techniques d'inscription) jusqu'aux objets intellectuels de la production et habiletés cognitives et culturelles de la réception, sans omettre les agents et les institutions de la conservation, puis de la transmission. Les pratiques cognitives, et culturelles au sens plus large, sont rangées dans le domaine de la réception.

5 The Domestication of the Savage Mind', la 'raison graphique', selon l'expression choisie comme titre de la traduction française (Paris, éd. de Minuit,1978), étudie les incidences de la 're-présentation' graphique du langage sur la formation des processus cognitifs en s'intéressant aux divers modes d'organisation des données par l'écriture: listes; graphiques; tableaux; index; cartes ... . Jack Goody dégageait, de la sorte, les conditions culturelles et historiques de la constitution d'une logique 'scripturale'(ou 'scripturalité') qui, par les effets contrôlés de l'écrit, conduisait à un nouveau rapport de soi au monde mis en œuvre à travers un outillage mental différent et, surtout, des procédures cognitives entièrement nouvelles. Les travaux menés sur le terrain, dans les territoires du nord Ghana, notamment sur la récitation du Bagré, avaient servi de base préparatoire à la définition de la 'raison graphique' à partir des observations réunies sur place, chez les LoDagaa ${ }^{9}$, et ouvert la voie à l'étude comparée des processus cognitifs au sein des sociétés avec et sans écriture. J'en retrouve la trace dans l'examen méthodique qui sert d'introduction à Representations and Contradictions, Ambivalence Towards Images, et dans certaines des prises de position bien affirmées, telle celle-ci :

« (...) Dans mon étude des images picturales, des icônes, je commence par l'Afrique, écrit Jack Goody, et suggère que certaines conceptions que nous trouvons explicitement exprimées non seulement en Europe mais aussi en Eurasie, et de manière on ne peut plus persistante en Asie de l'ouest se retrouvent peut-être en Afrique dans un état implicite et embryonnaire. J'essaierai d'en donner des preuves, mais elles sont maigres et il n'est pas facile de cerner des croyances qui, explicites dans maintes cultures écrites, sont implicites dans les cultures orales. L'inégale distribution des sujets imagés et des cultures imagières (imaging), c'est-à-dire leur absence dans certaines sociétés et leur présence dans d'autres (en Afrique comme en Eurasie), me paraît suggérer des problèmes parallèles quant à la nature des représentations. $»^{10}$

Et d'ajouter :

« (...) Cette distribution est imputée non pas à la culture - la plus faible de toutes les explications possibles - , mais à une ambivalence à l'œuvre dans la conceptualisation même des 'images'. " ${ }^{11}$ 
7 A une simple approche culturelle, ou culturaliste, est substituée une analyse structurelle des processus cognitifs à l'œuvre dans une société sans écriture dont la 'maigreur' de la culture imagière révèle bien plutôt les tensions internes comme le pouvoir d'autocritique, qu'un trait de culture ${ }^{12}$.

«L'élément cognitif est d'une importance capitale, surtout pour rendre compte des changements et des différences, tandis que la référence à des correspondances symboliques profondément enracinées conduit à postuler une structure statique. $»^{13}$

8 The Logic of Writing and the Organization of Society ${ }^{14}$ prend en compte les principaux aspects de l'organisation du discours écrit au sein d'une société : l'autonomisation du discours religieux fixé par écrit; l'émergence puis la consolidation de la bureaucratie administrative et politique; l'invention du calcul dans l'univers de l'économie marchande et bancaire ; enfin, la codification écrite des normes juridiques. Toutes les orientations ainsi dessinées proviennent de l'ouvrage précédent sur la domestication de la pensée sauvage, et ont été reprises ultérieurement ${ }^{15}$. La langue écrite est l'un des moyens, de loin le plus conséquent selon Jack Goody, pour la production du social et du cognitif, et pas seulement un code très élaboré, ni même sophistiqué.

The Interface between the Written and the Oral ${ }^{16}$ réexamine l'impact des premières formes d'écriture sur les littératures orales antiques, le jeu des interactions entre les cultures, orales et écrites en Afrique occidentale contemporaine, particulièrement, et surtout les enjeux de la connaissance et de la domination liés à la maîtrise des médiations écrites et à leurs apprentissages initiaux. L'étude du Bagré, ainsi que de ses modes de récitation chez les LoDagaa du nord Ghana, importe aussi à la compréhension de tous les mécanismes inscrits dans le champ des médiations, ceux relatifs aux modalités de la transmission comme ceux de la 'canonisation' du patrimoine immatériel ${ }^{17}$.

L'apport du livre, paru en 1997, sur la peur des re-présentations et l'ambivalence des attitudes à l'égard des images se place à la suite de toutes ces analyses, comme un jalon de plus dans l'enquête sur les fonctionnements des processus cognitifs au sein des sociétés.

« S'il en va ainsi, l'ambivalence et les contradictions cognitives à l'égard des représentations se retrouveraient dans la gamme des sociétés humaines, pas nécessairement dans toutes, mais les réponses seraient différenciées selon la nature du mode de communication. Tel est le thème que j'entends explorer. $»^{18}$

11 Etant admis que la 'littératie' est un système structuré, et structurant, des rapports au monde, étant posé qu'elle se manifeste également en dehors des seules pratiques langagières, ainsi que dans l'univers connexe de la 'communication graphique' ${ }^{19}$, il est possible d'analyser les principaux aspects des cultures imagières, comme des cultures ouvertes aux fleurs, en fonction des processus de connaissance propres aux sociétés humaines.

\section{Le processus de re-présentation et les contradictions cognitives}

12 A partir des observations faites sur l'absence des images, et non leur présence, en tenant compte dès lors de leur inégale distribution dans l'espace et dans le temps (phases chronologiques), Jack Goody compare entre elles plusieurs aires géographiques en essayant, toujours, de distinguer les différents régimes d'historicité possible ${ }^{20}$. Il 
reprend sur le fond les interrogations, et la méthode, qui ont été les siennes dans trois de ses ouvrages publiés peu auparavant: Cooking, Cuisine and Class: A Study in Comparative Sociology (1984); The Culture of Flowers (1993) ; The East in the West (1996) ${ }^{21}$.

\section{Examen des questions posées} larges similitudes apparaissent entre les grandes sociétés d'Asie et d'Europe, distinguées de celles de l'Afrique noire. Pour les cultures imagières de par le monde, le partage est moins tranché et s'établit entre 'formes culturelles épaisses' et 'formes culturelles maigres', indifféremment ici ou là, mais suivant des séquences temporelles différentes, qui incluent justement des comparaisons possibles entre l'Eurasie et l'Afrique noire. De plus, ce qui ressort encore de l'étude, c'est une inégale distribution des sujets imagés et des cultures imagières, en Eurasie comme en Afrique, donc des questions concomitantes posées sur la nature des re-présentations ${ }^{22}$. C'est aussi que l'inégale distribution n'est pas imputable à la culture, mais à l'ambivalence d'attitudes à l'œuvre dans la manière de concevoir, puis de faire, enfin de voir les images. L'argument intéresse alors directement la discussion des «modes de pensée », dont certains aspects ont des caractères liés aux formes de développement, aux modes de communication et de production. Il ne suffit pas, en effet, de placer les sociétés les unes à côté des autres de façon relativiste ; il faut surtout se demander comment les sociétés passent d'un mode à l'autre, car autrement on ne pourrait comprendre ni le passé eurasien ni le présent africain. Pour Jack Goody,

«(...) des traits cognitifs généralisés de la situation humaine donnent naissance à des ambiguïtés, à des ambivalences et donc à des doutes, se soldant par des manières diverses d'affronter ce problème selon les individus et les sociétés. (...) $»^{23}$

Le fond du problème reste l'intelligence humaine générale des diverses possibilités qu'offrent des situations ambivalentes de connaissance. Jack Goody évoque les rapports problématiques entre le " changement » et les « dilemmes cognitifs». En revenant sur l'exemple du luxe, il rappelle comment les «contradictions impliquées par l'usage d'articles de luxe » dans des situations de pauvreté, voire de pénurie, créaient chez certains un problème cognitif potentiel. Autrement dit, la contradiction sous-jacente reconnue par tous les participants donnait naissance à un autre type d'appréciation de ces activités, donc à une ambivalence très forte dans la perception de celle-ci. Cependant, l'expression du problème posé en termes de 'contradictions cognitives' l'inscrit au plan sociétal, d'emblée, dans les usages du langage et, au-delà, dans la définition de l'humain : en effet, toutes les objections aux re-présentations découlent de ces pratiques, donc du concept de la 'littératie' suivant Jack Goody.

«(...) Pour moi, au contraire, écrit-il, les problèmes liés à la représentation ne sont pas inscrits en nous (wired), mais naissent des contradictions cognitives de la vie humaine et ne deviennent explicites que lorsqu'une langue écrite rend cette discussion inévitable en objectivant la pensée de façon particulière. (...) $»^{24}$

D'où une différence importante entre les sociétés avec ou sans écriture, parce que « (...) l'écriture ajoute une dimension particulière. "

Dans ce contexte, quels sens donner au mot «re-présentation »? Certainement pas le sens de représentation politique d'un homme par un autre, ainsi que des historiens de l'Antiquité pourraient très bien le suggérer avec justesse ${ }^{25}$. Jack Goody l'emploie au sens littéral du latin « re-présenter »; en effet

Bulletin du centre d'études médiévales d'Auxerre | BUCEMA, Histoire de l'art \& Anthropologie 
«(..) mettre en présence de quelque chose qui était précédemment absent; pas simplement d'incarnation d'une abstraction dans un objet, mais de présentation de quelque chose d'une manière différente, comme dans la peinture d'un chien. Elle a presque toujours un aspect visuel, puisque ainsi va le monde, mais elle inclut la représentation d'abstractions, comme le courage, évoquées plus haut. En fait, le concept même de courage est une re-présentation d'autre chose. Voici pour l'extension de l'idée. (...) $»^{26}$

17 En suivant Ernst Gombrich (1909-2001), Jack Goody ne relie pas du tout 'représentation' et mimétisme, plutôt que réalisme, même s'il suppose souvent que la 'présentation' de la réalité implique une certaine forme de correspondance : ce n'est pas l'exactitude qui distingue la 're-présentation' parce que celle-ci n'est jamais une réplique, mais qu'elle renvoie à l'original sans jamais le copier. De même, et à la différence de Carlo Ginzburg, la 're-présentation' ne saurait être réduite, selon Goody, à la seule praesentia des historiens et, plus largement, des praticiens dans le champ des sciences sociales et humaines ${ }^{27}$. Ce qui l'emporte à ses yeux, indépendamment même de la définition analytique de la re-présentation, c'est la question de la perception souvent changeante qu'a l'acteur de ce qui est présenté et de ce qui est re-présenté. En employant le vocabulaire qui est le sien, celui d'un anthropologue, et donc en ayant soin de distinguer l'acteur de l'observateur, Jack Goody rejoint partiellement les positions d'Ernst Gombrich sur l'image et la perception; plus largement, sur le processus de re-présentation impliquant celui qui voit et qui agit ensuite, ou est agi par ce qu'il a vu' ${ }^{28}$.

Pour Jack Goody, ce processus est essentiel à la société humaine, par et dans le langage, mais comporte toujours un aspect négatif en ce que ce qui est re-présenté n'est jamais ce qui est présenté de prime abord. L'absence de l'objet peut donner naissance à d'autres questions, à des remises en cause, à des contestations. Le mot cheval n'est pas un cheval, l'animal, mais son absence. Or, selon lui, cette situation suscite de fortes contradictions cognitives relatives aux images, d'autant plus fortes quand nous avons affaire à des images de la divinité, notamment à celles du Créateur qui mettent en jeu l'unicité du plan de la création.

«Il existe cependant un problème touchant la manière de s'adresser à Dieu, mais aussi de le représenter. A la différence d'autres entités, le Dieu suprême n'a pas d'image figurative en raison des doutes entourant la représentation du Créateur par le créé, de l'immatériel par le matériel. Comment peut-on recréer le Créateur, en particulier sous une forme plastique, et surtout sous une forme tridimensionnelle où l'effort pour atteindre la réalité est plus évident? Le problème paraît parfois s'appliquer, quoique avec moins de rigueur, aux autres créatures divines, et, dans l'islam et le judaïsme, à tout ce que le Créateur a créé. Car un homme qui crée une image répète Son acte unique de Création. Cette idée d'unicité de la Création occupe une place centrale dans de nombreuses cosmologies africaines tandis que certaines actions humaines paraissent la remettre en cause. $»^{29}$

Relèvent de cette catégorie de 're-présentation' les reliques, le théâtre, la fiction, le mythe et, aussi, quoique dans un domaine adjacent, le sexe, puisqu'ici la question touche avant tout à l'auto-représentation et aux raisons pour lesquelles nous nous dérobons à lui. Il y a 'contradiction' quand une connaissance est contredite par les événements ou par les faits, voire les deux ensemble. Les contradictions cognitives sont impliquées dans, et par, le processus même de la connaissance; elles ont trait à la compréhension humaine du monde ${ }^{30}$. Ainsi comprise, l'expression de 'contradiction cognitive' ne serait guère éloignée, d'après Jack Goody, de celle de «dissonance 
cognitive » qu'utilise le psychologue Festinger, et nécessite donc des remises en situations concrètes.

\section{Situations}

20 Elles sont au nombre de deux pour les cas étudiés dans le domaine des images, que ce soit l'absence d'images ou leur rareté: d'une part, l'Afrique, et l'exemple particulier du nord Ghana; d'autre part, l'Eurasie et les vagues d'iconoclasme successives, depuis l'Antiquité chrétienne jusqu'à l'époque des Réformes, et au-delà encore.

21 En Afrique, la situation est variée et complexe. Au premier aperçu, d'après les objets exposés ou montrés dans les vitrines des musées, masques et figures sculptées abondent. L'ensemble des traits généraux des cultures imagières permettent de supposer une forte 're-présentation' des objets ${ }^{31}$, et selon certains l'indice d'un faible niveau de spiritualité, à tout le moins celui d'une relation trop concrète avec le divin, trop souvent rendu alors de manière anthropomorphique ou zoomorphique. Pourtant, Jack Goody, et d'autres avec lui, notent qu'on ne trouve d'images figuratives que par intermittence; que les autels peuvent avoir une forme figurative aussi naturellement qu'une forme non figurative ou abstraite ${ }^{32}$; qu'il ne peut y avoir aucune forme, par exemple, le dieu suprême des indigènes, le High God des Anglo-Saxons, qui ne prend que rarement, sinon jamais, une forme figurative : en ce cas, il n'y a ni re-présentation, ni image, ni icône et, à de très rares exceptions près, on ne lui donne ni autel, ni centre de culte matérialisé. Au début du XVIIIe siècle, un courtier hollandais, un certain Bosman, observait que

«Dieu étant invisible, ils disent qu'il serait absurde d'en faire une quelconque

représentation corporelle. $»^{33}$

On ne trouve de re-présentations que des divinités secondaires qui sont les intermédiaires entre Dieu et l'homme. Dans ces autres dieux, entre autres pour la religion des Nuer, Edward-Evan Evans-Pritchard (1902-1973) proposait de voir des « réfractions » de l'esprit ou du Dieu suprême, même s'il sous-estimait ainsi leur statut individuel : pour la plupart des membres des Nuer, en effet, ces autres dieux sont les agents les plus importants de leur vie spirituelle, aussi importants voire plus, toutes proportions gardées, que les saints patrons du catholicisme populaire sur les rives nord de la Méditerranée. Ces "réfractions» reçoivent constamment des sacrifices. Par contre, dans les pratiques locales, rares sont les moyens de communiquer avec Dieu, soit verbalement, soit par des dons, puisqu'il n'y a ni sanctuaire ni autel. Jack Goody l'avait observé, de son côté, chez les LoDagaa, dans un conte où le lièvre déjoue les menées de Dieu à seule fin d'être à son tour circonvenu par la perdrix, l'histoire s'achevant par cette question: "Comment une créature peut-elle être plus sage que le Créateur?»

A partir d'exemples repris aux anthropologues, ou de ses propres recherches sur le terrain, Jack Goody suggère que l'ambivalence africaine envers le Dieu unique est, à plus d'un titre, exemplaire de quelques-unes des contradictions nécessaires qui surgissent quand on s'applique à concevoir l'immatériel. Pourquoi les religions africaines manifestent-elles cette réticence à se servir d'images pour essayer de rendre visible le Dieu créateur, de même qu'à l'approcher fût-ce avec des mots? Pour lui, une partie de la réponse, mais une partie seulement, réside dans le problème du mal. L'acte 
de création, qui a fait de l'univers la demeure de l'homme, n'est pas un bien sans mélange : le mal a pénétré le monde et sa présence doit être expliquée ${ }^{34}$.

Des raisons d'ordre 'général' peuvent encore être évoquées, en relation plus étroite avec la question abordée. D'après l'enquête conduite chez les LoDagaa, Jack Goody est-il fondé à supposer un problème autour de la 're-présentation' ? Il peut suggérer, d'abord, qu'une contradiction d'ordre de la connaissance y est ressentie entre divinité et sanctuaire («fétiche»?), immatériel et matériel. Il peut aller plus loin et formuler l'hypothèse d'une contestation implicite de la relation, mais aussi imaginer qu'à un moment donné, à l'instar de ce qui se passe dans les cultures écrites, les LoDagaa ont en effet parlé de cette situation de conflit, sinon d'épreuve, qu'ils l'ont choisie ou, inversement, qu'ils ont décidé de ne pas fabriquer d'icônes (au moins dans certains contextes). Jack Goody pense que des problèmes d'ordre cognitif ne sont pas limités aux héritiers des cultures du Proche-Orient, aux utilisateurs des textes écrits et aux cultures de la 'littératie', mais qu'ils surgissent également, de manière peut-être moins évidente, dans les cultures orales. Cette hypothèse émise sur les icônes est renforcée quand il tient compte du contexte des 're-présentations' figurées interdites : celui du Dieu suprême; celui des divinités sérieuses. Dans ces deux cas, les LoDagaa "préfèrent » des formes non figuratives qui suscitent beaucoup moins de difficultés ${ }^{35}$.

L'enseignement est clair du double point de vue de l'absence des icônes ou des images et de leur inégale répartition ${ }^{36}$. En effet, l'existence de ces questions, explicites ou implicites, peut conduire tel groupe à choisir une ligne d'action, tel autre à en choisir une autre, chacune pouvant changer ensuite avec le temps : ce sont là des alternatives inscrites dans les contradictions cognitives de la situation, et elles contribuent à expliquer la distribution, dans des régions très voisines parfois, de formes abstraites et d'images figuratives, comme les sanctuaires consacrés aux ancêtres ou aux divinités, mais aussi l'accent différent que mettent ces sociétés sur toutes les 're-présentations' imagées. A plus long terme, du fait de ces notions implicites, les injonctions islamiques et chrétiennes de détruire les images des anciens dieux ont pu toucher une corde sensible dans la pensée des indigènes et leur devenir ainsi plus acceptables qu'on ne le pensait à première vue: en d'autres termes, l'ambivalence qui préexistait et qui coexistait dans leurs modes de pensée sur le divin, puis sur ses images en second lieu, a pu miner leur degré de résistance par rapport à ces injonctions le jour où elles leur furent imposées ${ }^{37}$. L'explication avancée vaut au-delà des cultures imagières de l'Afrique de l'Ouest, et de l'exemple des LoDagaa et s'appliquerait aussi aux situations de rencontres conceptuelles en Eurasie, comme celle qui eut lieu dans l'Empire byzantin, entre IIIe et IXe siècle, pour adopter une chronologie d'ensemble, à l'intérieur des provinces orientales au contact avec l'Islam ${ }^{38}$.

Sans nous arrêter davantage à la querelle des images dans le monde byzantin aux VIIIe et IXe siècles, autrement dit à l'iconoclasme entendu au sens strict, c'est-à-dire en suivant l'approche de Jack Goody, sans privilégier l'événementiel et le particulier, j'examinerai l'autre mise en situation, celle de l'Eurasie. Pour la démonstration, il tient compte de plusieurs indicateurs, bien étudiés par ailleurs, se rapportant tous au corps mort, à ce qu'il en subsiste de traces, de vestiges, et aux utilisations qui peuvent en avoir été faites: pèlerinages; miracles; reliques. Chacun révèle le même genre d'ambivalences mentales autour des difficultés qu'il y a à concevoir ou à imaginer l'immortel à partir du mortel, à s'intéresser à la dépouille corporelle qui, tout à la fois, attire du fait de son pouvoir (personnage d'autorité ou personnage saint) mais aussi 
repousse par suite de la perte de ce pouvoir dans la mort. De façon générale, le concept de ce qui reste, ou de relique, est caractérisé par l'attrait mais aussi la répulsion, qui s'attache aux morts, mais encore par la prise de distance inévitablement que la mort suscite toujours. Le culte des restes corporels, que le christianisme favorisa, est alors riche en tensions, critiques et en 'contradictions cognitives': les reliques sont des objets normalement considérés comme impurs - chair morte, ossements, parties du corps -, et néanmoins vénérés comme saints ${ }^{39}$. C'est le cas 'à part' d'êtres qui ont été humains mais qui, maintenant, sont spiritualisés et considérés comme des saints, comme des agents surnaturels (selon Peter Brown, que Jack Goody cite dans tout son chapitre 3 et qu'il commente avec $\operatorname{soin}^{40}$ ). Entre ces deux aspects, qui apparaissent comme les deux versants d'une seule et même structure, les contradictions sont profondes et, pourtant, présentes ensemble partout.

«Dans l'au-delà, en effet, les objets matériels sont clairement opposés à une existence spirituelle (immatérielle). Cette opposition était particulièrement marquée lorsque l'objet matériel était déplacé d'un endroit à un autre, était exhumé pour être transporté ailleurs, ou même volé par ceux qui le convoitaient comme une source de pouvoir et de richesse. $»^{41}$

Plus que la prolifération des reliques et les propos critiques qu'elle entraîna, Jack Goody souligne les problèmes cognitifs liés au culte des reliques, à la 're-présentation' du corps glorieux à partir des restes, peu glorieux, du corps mort. Si pour Hans Belting, l'absence fonde l'image, pour Jack Goody elle institue un paradoxe vivant, entretenu avec soin par l'Eglise, car la croissance du culte des reliques va de pair avec l'expansion universelle (catholique) de celle-ci. Demeure le paradoxe qui est le signede ces contradictions à l'œuvre dans la connaissance du divin et dans la manière de le concevoir pour mieux l'approcher.

«(...) Le paradoxe consistant à attribuer un rôle actif aux restes matériels des morts tout en rejetant l'essence spirituelle dans un autre royaume, les problèmes posés par les atteintes à la séparation entre les vivants et les morts, le matériel et l'immatériel, le paradoxe de l'inhumation d'un humain et de l'exhumation des os sont autant d'éléments que l'on semble retrouver derrière l'acceptation générale de l'utilisation de reliques corporelles par certains et de leur rejet par d'autres. (...) »

Et d'ajouter :

«(...) On constate, une fois encore, que ce qui est explicitement formulé dans les cultures de l'écriture l'est implicitement dans les cultures orales; de façons différentes, les unes et les autres attirent l'attention sur des contradictions cognitives entre l'évidente mortalité du corps et les idées sur l'immortalité de l'âme. C'est le paradoxe des dépouilles mortelles et des désirs d'immortalité. $\$^{42}$

Paradoxe des défis également.

\section{Pertinence des concepts dans le champ de l'iconologie aujourd'hui}

30 Je distinguerai deux niveaux de pertinence : interne à l'analyse de Jack Goody lui-même et à son approche conceptuelle; externe par rapport à ses conclusions et à leur élargissement dans tout le champ des travaux sur l'image, la «culture de l'imago » (Jean-Claude Schmitt), qui s'inscrit comme un pendant de la 'culture imagière' telle que l'analyse Jack Goody. Enfin, je proposerai quelques notions complémentaires pour tenter de mieux cerner le champ d'études tel qu'il s'est peu à peu configuré, à l'articulation de l'iconographie et de l'iconologie. 
31 - Dans l'approche même de Jack Goody, la pertinence me paraît devoir être soulignée à un double point de vue.

Il parvient, d'abord, à étendre à l'iconique l'étude des problèmes d'ordre cognitif qui traversent, selon lui, tout le champ de la 'littératie'. Son étude porte sur quatre ressorts fondamentaux qui font voir ce qu'est l'image religieuse qui 're-présente' ${ }^{43}$ :

- la création même d'images, de "ressemblances », peut sembler reproduire l'acte unique de la création ;

- la création d'images religieuses soulève une question spécifique que Goody a pointée, à plusieurs occurrences, dans sa démonstration: comment traduire l'élément immatériel, voire spirituel, sous une forme humaine, ou quelle qu'autre forme que ce soit?

- la situation à part du Dieu suprême, le problème posé par la 're-présentation' non pas de la création, mais du Dieu créateur lui-même

- la tendance générale, enfin, à se méfier des images, parce que ce sont des 're-présentations' trompeuses des réalités.

Rejeter les images, l'iconophobie, ne consiste pas seulement à refuser les images des faux dieux. Elle comporte aussi une dimension philosophique en ce que toute image de la divinité, toutes les images religieuses et, parfois, les images de tout ce que la divinité a créé, ou même, les images que l'humanité a forgées, sont à abandonner. Ce sont des tendances largement répandues parmi les cultures humaines même si, souvent, elles sont en état de "latence ${ }^{44}$. Une solution au problème, posé de la sorte, consiste à éviter toute image de la divinité et à s'appuyer uniquement sur le mot : d'où en Israël, l'accent mis sur la parole de Dieu; de même, chez les premiers chrétiens, en islam, chez les protestants et dans le bouddhisme zen, toutes religions pour lesquelles la parole est diamétralement opposée à l'image. Sur l'autel, dans le temple, il n'y a pas de figuration, et les seules offrandes sont réduites aux mots, aux prières et aux hymnes, le cas échéant sans aucun accompagnement musical ${ }^{45}$. En Afrique, si je reviens à cette mise en situation, dans ses travaux antérieurs, Jack Goody a montré que les chrétiens et les musulmans étaient connus, d'abord, comme ceux (ou celles) qui priaient, par opposition à ceux qui faisaient des offrandes d'autres espèces, des sacrifices sanglants notamment. Ces offrandes matérielles impliquaient, par ailleurs, une série de contradictions qui se retrouvent au cœur du processus de 're-présentation', tel que Jack Goody l'a étudié ${ }^{46}$ : sur un plan matériel, en effet, le paradoxe du sacrifice est que les dons ne sont pas consommés toujours de la manière profane courante, mais distribués aux donateurs, aux fidèles, ou abandonnés ; ce paradoxe peut être levé en déplaçant le don au niveau de la parole ou, inversement, en offrant l'âme immatérielle du don ou en laissant un élément vital, le sang ou la libation, se répandre sur la terre, ou pénétrer en ses profondeurs. Le paradoxe du sacrifice provient de la manière dont on conçoit la divinité : non humaine, mais possédant des attributs humains. La question se pose avec la même ambivalence pour l'image ou pour l'icône.

Il précise, ensuite, les rapports intrinsèques entre 're-présentation' et 'contradictions cognitives', à l'intérieur du processus :

«(...) Ce processus vient de la présence de contradictions cognitives, d'ambiguïtés potentielles de la condition humaine qui dérivent du contexte interactif et qui font elles-mêmes partie de la culture transmise. Autrement dit, la culture ne consiste pas simplement en tendances innées ou en procédures coutumières (traditionnelles) d'une espèce socialisée, mais elle contient aussi un noyau de doute, sa propre auto-critique susceptible de déboucher sur des formes de comportement 
opposées. C'est précisément ce qui arrive dans de nombreux exemples de représentation. (...) $»^{47}$ observé à partir des contradictions dans le champ de la connaissance s'avère patente par rapport à la 'culture de l'imago' particulière à l'Occident médiéval. Ce sont peut-être les travaux de Jean-Claude Schmitt qui, pour la France du moins, ont ouvert et précisé le mieux, en ces termes, le champ de l'enquête, très proche au fond de celui qu'expérimente, en anthropologue, Jack Goody, mais en restant pour sa part un historien de l'image ${ }^{49}$. S'interrogeant sur cette culture, et ses évolutions, entre le VIIIe et le XIIIe siècle, il se montre attentif aux facteurs internes de la transformation dans les images religieuses, pour l'essentiel les contestations formulées par les hérétiques dans leurs critiques des images : à ce sujet, le point de rupture est bien le début du XIe siècle, date charnière au centre de toute la conjoncture ${ }^{50}$. En s'attachant à la biographie reconstituée d'Hermann le juif, cette fois en historien soucieux des textes, Jean-Claude Schmitt rejoint encore tout à fait les analyses de Jack Goody sur les contradictions cognitives à l'œuvre dans un destin individuel et l'ambivalence foncière de toute attitude par rapport à la vie. En même temps qu'au christianisme, Hermann-Judas se convertit à l'image et à ses pouvoirs de suggestion, dans le courant du premier tiers du XIIe siècle ${ }^{51}$ et, de la sorte, rencontre les arguments qu'avançait, entre autres, un Rupert de Deutz (1075-1129), notamment dans son traité Anulus sive Dialogus inter Christianum et Iudaeum, écrit en 1126 à la demande de Rodolphe de Saint-Trond, abbé de Saint-Pantaléon de Cologne. Les traits génériques de la conception des images chrétiennes étaient, selon Rupert, les suivants :

- les images remontent à la longue tradition de la décoration du Temple à la louange de Yahvé ;

- il y a une continuité de l'histoire des images, de même qu'il y a une continuité de l'histoire des serviteurs de Dieu, depuis les patriarches jusqu'aux saints martyrs de l'Eglise ;

- trois types caractérisent les modes de figuration : l'ornemental dans le sanctuaire pour la louange visuelle de Dieu; les memorabilia, les images narratives, qui rappellent les gesta des principaux acteurs de l'Histoire sainte ; enfin, à part, en raison de son éclat et de son mode de justification - l'Incarnation -, l'image du Christ crucifié qui évolue à partir du XIe siècle, et surtout au XIIe siècle, vers une 're-présentation' tridimensionnelle ${ }^{52}$.

Dans le repérage des traits génériques comme dans la compréhension de la 'culture imagière' des VIIIe-XIIIe siècles en Occident, la pertinence des concepts développés par Jack Goody est totalement opératoire et renouvelle les rapports entre l'anthropologie et l'approche des images dans l'histoire. Pour compléter la lecture du livre de Jack Goody, et dans la suite des réflexions sur les rapprochements possibles avec l'imago, je formulerai l'hypothèse complémentaire de deux autres notions qui s'ajoutent à la définition de la 'culture imagière' telle que l'analyse l'a précisée peu à peu : ce sont les notions d'image intensive' et de 'reste' ou d"image excroissante'. Par image intensive, je voudrais souligner la prégnance des relations au corps, et surtout au corps absent, et 
aménager ainsi un espace de discussion autour des pratiques rituelles qui viennent accompagner tout le processus, mais aussi le constituer et l'achever. Pour l'Occident aux VIIIe-XIIIe siècles, ce sont tous les développements de la liturgie, fondamentaux dans la prise en compte des images dans l'histoire, qui imposent en même temps la production d'une symétrie visuelle, entre ce qu'on voit autour des images et les images elles-mêmes, et renforcent l'unicité du processus de 're-présentation' dans un lieu défini alors comme un "lieu d'images »: par exemple,au XIIe siècle, dans les décors peints des églises du Berry comme à travers les images hagiographiques de SaintEutrope aux Salles-Lavauguyon; dans les Abruzzes, sur les murs de la petite église de Bominaco, vers 1263 ; à l'intérieur de la Chapelle dite de l'Arena, à Padoue, en $1305{ }^{53}$. Par image excroissante, ce sont les relations verticales et horizontales que tout le processus de la 're-présentation' entretient forcément avec ce qui l'entoure : relations verticales, je désigne par ce terme la filiation positive qui réunit l'image à son modèle, quel que soit le nom qu'on veut lui donner, et donc sa place dans la généalogie que forme tout discours graphique; relations horizontales, j'entends par là tous les dispositifs d'ancrage dans un territoire, plus ou moins délimité, mais visible, parce que matérialisé, comme rendu tangible au moyen des matières et des formes utilisées par les images, pour l'expression des liens forts qui se sont tissés entre les hommes et le divin. De l'entrecroisement de ces deux directions, je dégagerai alors la notion de reste, c'est-à-dire l'ensemble des marquages et des signes graphiques produits par l'image dans le processus même de sa 're-présentation', de même que l'ensemble de ses relations avec les supports matériels. Il en résulte un surplus de valeur qui provient de l'image elle-même et contribue à lui rendre toute sa force ${ }^{54}$.

C'est bien en ces points de l'analyse que doit être repensée, puis reformulée, l'articulation toujours problématique de l'iconographie à l'iconologie, et pleinement réévaluée l'approche de Jack Goody en anthropologie cognitive.

\section{NOTES}

1. Ce texte est la première version d'une étude présentée dans le cadre du Clark - INHA Workshop, Iconography and Iconology Today, (19-25 août 2007), sous l'intitulé Icons, Iconoclasm and Religions. Reading: Jack GOODY, Representations and Contradictions. Ambivalence Towards Images: Theatre, Fiction, Relics and Sexuality, Londres, Blackwell Publishers, 1997 (trad. fr. 2003, Paris, La Découverte, Pierre-Emmanuel DAUZAT). Je tiens à remercier particulièrement les organisateurs de cette rencontre Michael-Ann HOLY, Mark LEDBURY, Olivier BONFAIT et Thierry DUFRÊNE, pour leurs remarques et discussions des arguments que j'avance.

2. Nelson GOODMAN, Ways of Worldmaking, Hackett Publishing Company, 1978, part. chap. 4.

3. Hans BELTING, "Bild und Tod», repris in ID., Bild-Anthropologie: Entwürfe für eine Bildwissenschaft, Munich, Verlag Wilhelm Fink, 2001.

4. Meyer SCHAPIRO (1904-1996), "On some problems in the Semiotic of Visual Art, Field and Vehicle in Image-Signs » in Semiotica 1, 3, (1969), p. 223-242 (texte d'une conférence prononcée en septembre 1966 à Kazimierz, en Pologne) ; définition du terme 'champ', Jean-Christophe BAILLY, Le champ mimétique, Paris, éd. du Seuil, 'La Librairie du XXIe siècle', 2005, part. p. 11-64. 
5. Michael Ann HOLLY, Panofsky and the Foundations of Art History, Ithaca/Londres, Cornell University Press, 1984, p. 21-45; p. 97-113 : pour une discussion d'ensemble de l'approche panofskyenne située par rapport aux principaux courants d'interprétation de son époque

6. Cambridge University Press, 1968.

7. En traduction française, par Jean-Claude LEJOSNE, in Pratiques 131-132, Metz, (déc. 2006), « 'La littératie'. Autour de Jack Goody », Mohamed KARA, Jean-Marie PRIVAT, dirs., p. 7-30 ; p. 31-68.

8. Cambridge University Press, 1977.

9. Jack GOODY, The Ethnography of the Northern Territories of the Gold Coast, West of the White Volta, Londres, Colonial Office mimeo, 1954; ID., «Religion and Ritual : The Definitional Problem » in British Journal of Sociology 12, (1961), p. 142-163 ; ID., The Myth of the Bagre, Oxford, Clarendon University Press, 1972.

10. Jack GOODY, op. cit., chap. 1, p. 28-29 (trad. fr.).

11. ID., op. cit., chap. 1, p. 29. Voir chap. 3, p. 110, pour le même genre de remarque.

12. Selon une approche comparable, voir Clifford GEERTZ, Bali. Interprétation d'une culture, recueil d'articles (1959-1973), trad. en fr. par Denise PAULME, Louis EVRARD, Paris, Gallimard, 'Bibliothèque des sciences humaines', 1983 ; ID., Local Knowledge. Further Essays in Interpretive Anthropology, New York, Basic Books, 1983 (trad. fr. par Denise PAULME, Paris, P.U.F., 'Sociologie d'aujourd'hui', 1986).

13. ID., op. cit., chap. 1, p. 30. C'est, du reste, la principale critique que Jack Goody formule contre les analyses de Victor TURNER et l'approche de «type particulariste ", ou historique, notamment dans son étude sur le culte des reliques; Jack GOODY, op. cit., p. 92-93. Pour une synthèse des positions de Turner, voir Victor W. TURNER, Edith L. B. TURNER, Image and Pilgrimage in Christian Culture, New York, Columbia University Press, 1978, p. 1-39 ; p. 140-171 ; p. 172-202.

14. Cambridge University Press, 1986.

15. En part. voir Jack GOODY, The Power of the Written Tradition, Washington/Londres, Smithsonian Institution Press, 2000.

16. Cambridge University Press, 1987.

17. Jack GOODY, The Power of the Written Tradition, op. cit., chap. 7 ; de manière plus générale, Canonization and Decanonization (Studies in the History of Religions), Arie van der KOOIJ, Karel van der TOORN, Leyde, Brill, 1998.

18. ID., Representations and Contradictions, Ambivalence Towards Images, op. cit., en part. chap. 1, p. 30 (trad. fr.).

19. Suivant le même ordre d'idées, Erwin Panofsky et, plus encore, Pierre Bourdieu (1930-2002) démontraient, par exemple, le second interprétant le premier, qu'un transfert des modes de la pensée écrite des scolastiques pouvait avoir donné forme au style de l'architecture gothique ; voir Philippe HAMON, « Texte et architecture », in Expositions, Paris, José Corti éd., 1989, p. 21-52 ; Jean-Marie PRIVAT, «Un habitus littératien?», in Pratiques, op. cit., p. 125-130. Précisément la réflexion engagée par Pierre Bourdieu sur le livre d'Erwin Panofsky n'était en rien 'particulariste', suivant le terme employé par Jack Goody, mais très largement an-historique. Sur cette question, souvent mal évaluée en France, Bruno REUDENBACH, «Panofsky et Suger de Saint-Denis ", in Histoire et théories de l'art. De Winckelmann à Panofsky, in Revue Germanique Internationale 2, 1994, p. 137-150.

20. Réflexions de ce type dans Marcel DETIENNE, Comparer l'incomparable, Paris, éd. du Seuil, 'La Librairie du XXe siècle', 2000, en part. chaps. 1 à 3.

21. Respectivement Paris, éd. du Centre Georges Pompidou, 1984 ; Cambridge University Press, 1993 et 1996.

22. Jack GOODY, «Icônes et iconoclasme en Afrique » in Annales E.S.C. 46, 6, (1991), p. 1235-1251, part. p. 1251: « (...) Malgré l'apparition fréquente de formes figuratives à trois dimensions, l'espace de leur distribution est loin d'être uniforme. C'est vrai des représentations en métal et, dans une certaine mesure, de la sculpture en bois plus fréquente dans les régions de forêt. Mais il 
y a des régions et des peuples où l'art figuratif n'apparaît pas du tout, ou seulement dans certains contextes restreints, ce qui laisse supposer que les enjeux sont plus importants qu'il n'y paraît. "

23. ID., Representations and Contradictions, Ambivalence Towards Images, op. cit., chap. 1, p. 29 (trad. fr.).

24. ID., Representations and Contradictions, Ambivalence Towards Images, op. cit., p. 41, p. 42 (trad. fr.).

25. Louis GERNET (1882-1962), Anthropologie de la Grèce antique, Paris, éd. François Maspero, 1968 ; rééd. Paris, Flammarion, 1982. Jean-Pierre VERNANT (1914-2006), «Image et apparence dans la théorie platonicienne de la mimesis » in Journal de Psychologie 6, 2, (1975), repris in ID., Religions, histoires, raisons, Paris, éd. La Découverte, 1979, p. 105-137. Réflexions d'ensemble, Paul VEYNE, «Buts de l'art, propagande et faste monarchique » in L'empire gréco-romain, Paris, éd. du Seuil, 'Des travaux', 2005, p. 379-418. Du point de vue de l'anthropologie des images et, plus particulièrement des rapports de l'image à la mort, Hans BELTING, «Bild und Tod " in BildAnthropologie, op. cit., pour la discussion (p. 214-217) de Jean-Pierre VERNANT, Figures, idoles, masques, Paris, éd. La Découverte, 1990.

26. Jack GOODY, Representations and Contradictions, Ambivalence Towards Images, op. cit., chap. 1, p. 44-46 (trad. fr.).

27. Carlo GINZBURG, « Représentation. Le mot, l'idée, la chose » in Annales E.S.C. 46, 6, (1991), p. 1219-1234 ; repris dans ID., Occhiali di legno. Nove riflessioni sulla distanza, Milan, Feltrinelli ed., 1998, p. 73-88 (trad. fr. par Pierre-Antoine FABRE, Paris, Gallimard, 'Bibliothèque des histoires', 2001, p. 73-88). Sur la notion de 'présentation', d'un point de vue anthropologique, Alfred GELL (1946-1997), Art and Agency. An Anthropological Theory, Oxford, Clarendon Press, 1998. Du point de vue matérielle des images, non plus de ce qu'elles signifient et de ce qu'elles « font ", mais de ce qu'elles sont et de ce qu'elles "veulent ", et en déplaçant le questionnement du pouvoir au désir, W. J. Thomas MITCHELL (1942- ), What Do Pictures Want?: The Lives and Loves of Images, Chicago/ Londres, University of Chicago Press, 2005, en part. p. 28-56, qui situe le problème de l'image dans le nœud formé par le désir, la demande, le besoin, en lieu et place des trois niveaux de l'analyse panofskyenne.

28. Michael FRIED (1939- ), Absorption and Theatricality, Chicago/Londres, University of Chicago Press, 1980, en part. p. 90-92.

29. Jack GOODY, Representations and Contradictions, Ambivalence Towards Images, op. cit., chap. 8, p. 278-279 (trad. fr.). Sur cette question fondamentale qui lie 'cosmothéisme', 'monothéisme', et image, voir les belles réflexions de Jan ASSMANN (1938- ), dont certaines traduites en français et publiées sous le titre Le prix du monothéisme, Paris, éd. Aubier, 'Coll. historique', 2007, en part. chap. 3.

30. Jack GOODY, Representations and Contradictions, Ambivalence Towards Images, op. cit., chap. 8. 31. Remarques très intéressantes dans Marc AUGE, Le dieu objet, Paris, éd. Flammarion, 'Nouvelle Bibliothèque scientifique', 1988. Voir toutes les données et les 'images' réunies dans Qu'est-ce qu'un corps? Afrique de l'Ouest, Europe occidentale, Nouvelle-Guinée, Amazonie, Catalogue d'exposition, 23 juin 2006-25 novembre 2007, dir. Stéphane BRETON, Musée du Quai Branly, Paris.

32. Qu'est-ce qu'un corps ?, op. cit., part. figs. 7 à 11, p. 30-34; notamment (p. 31) : « (...) Les autels du culte des ancêtres sont composés d'éléments d'où la figuration est absente ou réduite à l'état d'esquisse. (...)»: autels dagara (Burkina Faso) ; autels des ancêtres féminins aussi, re-présentés par de petits tabourets à quatre pieds; autels dogon; autels kassena (Burkina Faso); autels batammaliba (Togo); (p. 32) : « (...) D'autres types d'autels dont le principe n'est pas cumulatif obéissent cependant aux mêmes caractéristiques: absence ou altération de la figuration et enchâssement de ses éléments dans la terre. (...)»

33. Cité par Jack GOODY, Representations and Contradictions, Ambivalence Towards Images, op. cit., chap. 2, p. 71 (trad. fr.), utilisant Willehlm BOSMAN, A New and 
Accurate Description of the Coast of Guinea, Divided into the Gold, the Slave, and the Ivory Coasts, (Londres, 1705), Londres, 1967.

34. Jack GOODY, Representations and Contradictions, Ambivalence Towards Images, op. cit., chap. 2, part. 'Doutes sur les icônes'. De façon large, Jack GOODY, L'homme, l'écriture et la mort. Entretiens avec Pierre-Emmanuel DAUZAT, Paris, Les Belles Lettres, 1996.

35. Ibidem, chap. 2, passim p. 78 (trad. fr.).

36. Jack Goody n'établit pas une distinction tranchée entre l'icône et l'image, comme bien des anthropologues de la connaissance, mais considère qu'il peut utiliser le terme d'icônes à partir d'un certain degré de répétition.

37. Ibidem, chap. 2, p. 79 (trad. fr.).

38. Gilbert DAGRON, Empereur et prêtre. Etude sur le césaropapisme byzantin, Paris, Gallimard, 'Bibliothèque des histoires', 1996; ID., Décrire et peindre. Essai sur le portrait iconique, Paris, Gallimard, 'Bibliothèque illustrée des histoires', 2007, selon une interrogation plus large qui rattache la question du refus des images à tout un pan de la réflexion philosophique antique. Pour une remise en situation des questions et une analyse de cas, Art médiéval de la Méditerranée, Daniel RUSSOcoord., in Revue de l'Art, n. sp., 158-4 (2007).

39. Ibidem, chap. 3, passim et, plus précisément, p. 103-104 (trad. fr.). Jack Goody utilise John S. STRONG, « Relics » in Encyclopaedia of Religion, vol. 12, New York, 1987, p. 281 sq.

40. Peter BROWN (1935- ), The Cult of the Saints: Its Rise and Function in Latin Christianity, Oxford, Clarendon Press, 1981 ; ID., Society and the Holy in Late Antiquity, New York, Faber and Faber, 1982 ; ID., Power and Persuasion in Late Antiquity. Towards a Christian Empire, The University of Wisconsin Press, 1992.

41. Jack GOODY, Representations and Contradictions, Ambivalence Towards Images, op. cit., p. 104 (trad. fr.), qui cite Patrick J. GEARY, Furta sacra: Thefts of Relics in the Central Middle Ages, Princeton, 1978, passim.

42. Ibidem, chap. 3, p. 111-112 (trad. fr.).

43. Jack GOODY, Representations and Contradictions, Ambivalence Towards Images, op. cit., chap. 3, p. 67 (trad. fr.).

44. Ce serait à rapprocher des remarques d'Alphonse DUPRONT, Du sacré. Croisades et pèlerinages. Images et langages, Paris, Gallimard, 'Bibliothèque des histoires', 1987, part. p. 100-143 ('L'image de religion'), distinguant trois 'fonctions', comme en écho aux trois niveaux de l'analyse panofskyenne, à savoir : une fonction de représentation; une fonction symbolique; une fonction théophanique. Voir aussi, à présent, Ph. BORGEAUD, Aux origines de l'histoire des religions, Paris, Seuil, 'La librairie du XXIe siècle', 2004.

45. Nicolas LOSSKY, Essai sur une théologie de la musique liturgique. Perspective orthodoxe, Paris, Cerf, 'Patrimoines Orthodoxie', 2003, au-delà même de l'orthodoxie et pour toute la réflexion sur le silence dans l'église, et par rapport à la définition ecclésiologique du lieu de culte chrétien.

46. Jack GOODY, «Sacrifice Among the LoDagaa and Elsewhere: A Comparative Comment on Implicit Questions and Explicit Rejections », in Systèmes de pensée en Afrique noire, Paris, E.P.H.E., Ve section, Cahier 5, 1981, p. 9-22.

47. Jack GOODY, Representations and Contradictions, Ambivalence Towards Images, op. cit., p. 279 (trad. fr.).

48. Voir les études de cas réunis dans Crises de l'image religieuse. Krisen religiöser kunst, Olivier CHRISTIN, Dario GAMBONI, dirs., Paris, Editions de la Maison des sciences de l'homme, 1999, et part. la présentation, p. 1-15 (16-32).

49. Jean-Claude SCHMITT, Jérôme BASCHET, dirs., L'image. Fonctions et usages des images dans l'Occident médiéval, (" Actes du $6^{\mathrm{e}}$ International Workshop on Medieval Societies», Erice, Sicile, 17-23 octobre 1992), Paris, Le Léopard d'or, 1996, part. p. 29-37 («Imago : de l'image à l'imaginaire »).

50. Jean-Claude SCHMITT, « L'Occident, Nicée II et les images du VIIIe au XIIIe siècle » in Nicée II, 787-1987. Douze siècles d'images religieuses, Paris, éd. du Cerf, 1987, p. 271-301, part. p. 286 sq. ; pour 
d'autres analyses et une vue d'ensemble, ID., Le corps, les rites, les rêves, le temps. Essais d'anthropologie médiévale, Paris, éd. Gallimard, 'Bibliothèque des Histoires', 2001.

51. ID., La conversion d'Hermann le juif. Autobiographie, histoire et fiction, Paris, éd. du Seuil, 'La Librairie du XXIe siècle', 2003, part. p. 143-178 ('La conversion à l'image').

52. Ibidem, p. 166-167 ; ID., " Translation d'image et transfert de pouvoir. Le crucifix de pierre de Waltham (Angleterre, XIe-XIIIe siècle)» in Les images dans les sociétés médiévales : pour une histoire comparée, Jean-Marie SANSTERRE, Jean-Claude SCHMITT, éds., Bruxelles/Rome, Bulletin de l'Institut historique belge de Rome 69, (1999), p. 245-264.

53. Eric PALAZZO, Liturgie et société au Moyen Âge, Paris, éd. Aubier, 'Collection historique', 2000. Pour les exemples des décors peints énumérés, voir Marcia KUPFER, Romanesque Wall Painting in Central France. The Politics of Narrative, NewHaven/Londres, Yale University Press, 1993 ; Cécile VOYER, Faire le ciel sur la terre. Les images hagiographiques et le décor peint de Saint-Eutrope aux SallesLavauguyon (12e siècle), Turnhout, Brepols éd., 2008 ; Jérôme BASCHET, Lieu sacré, lieu d'images. Les fresques de Bominaco (Abruzzes, 1263) : thèmes, parcours, fonctions, Paris/Rome, La Découverte-Ecole française de Rome, 'Images à l'appui' 5, 1991 ; Laurent JACOBUS, Giotto and the Arena Chapel: Art, Architecture and Experience, Londres, Harvey Miller Publishers, 2007.

54. Sur cette question des rapports que l'image suscite elle-même, W. J. Thomas MITCHELL (1942- ), What Do Pictures Want? The Lives and Loves of Images, Chicago/Londres, The University of Chicago Press, 2005, part. p. 76-106. Pour les capacités d' " excroissance " de l'image, à partir d'études de cas, Landscape and Power, W. J. Thomas MITCHELL, éd., Landscape and Power, Chicago/ Londres, The University of Chicago Press, 1994 (2 éd., 2002).

\section{INDEX}

Mots-clés : iconologie, anthropologie, Goody Jack, représentation

\section{AUTEUR}

\section{DANIEL RUSSO}

Professeur à l'Université de Bourgogne, Directeur d'ARTeHIS-UMR 5594, Membre senior de l'Institut universitaire de France 